\title{
RELATÓRIO DA GESTÃO 2011
}

\author{
Silvana Calvo Tuleski \\ Marta Eliane Echeverria Borges ${ }^{\#}$
}

O presente relatório objetiva descrever a gestão editorial da revista Psicologia em Estudo no ano de 2011. É sempre importante iniciar contando um pouco da história deste periódico, com o objetivo de informar o leitor quanto ao seu percurso e aos percalços por ele enfrentados ao longo destes quinze anos de existência.

Se atualmente a revista tem periodicidade trimestral e conta com dezesseis volumes publicados, contabilizados a partir de sua origem em 1996, quando veio a público o Vol. 1, n. 1, sob editoração geral da professora doutora Maria Lúcia Boarini, do Departamento de Psicologia da Universidade Estadual de Maringá, é importante destacar que a periodicidade e a quantidade de artigos publicados a cada número foram crescendo ao longo destes anos.

Para retratar brevemente o seu crescimento, destaca-se o fato de que em seus primeiros cinco anos de existência a revista publicava, em média, dez artigos por número, com periodicidade semestral. A partir de 2001 este quadro começou a se alterar, com o aumento do número de artigos enviados para o periódico, que lhe permitiu cada vez maior visibilidade em nível nacional e internacional, ainda sob a editoração de sua fundadora.

Desde o seu início, o apoio e parceria em infraestrutura para parte do processo editorial, preparação, distribuição e indexação dos volumes, adveio da Universidade Estadual de Maringá, do
Departamento de Psicologia e, em 2008, do Programa de Pós-Graduação em Psicologia da mesma instituição.

A partir de 2006 a revista passou a ter como editora geral a professora doutora Rozilda das Neves Alves, que administrou o periódico até 2008, quando então assumiu a professora doutora Silvana Calvo Tuleski, a qual permaneceu como gestora até 2011. É importante destacar que a sucessão de editores tem como objetivo possibilitar a outros professores pesquisadores a experiência com a administração de um periódico científico de referência nacional como este. Sendo assim, terminada a gestão de três anos, a partir de 2012 assume como editor geral o professor doutor Paulo José da Costa.

Além das sucessões ocorridas referentes ao trabalho de editoria geral entre os triênios de 2006 a 2008 e de 2009 e 2010, houve também alterações na equipe da revista, decorrentes de dois fatores: perspectiva de aposentadoria de alguns professores e afastamento para pósdoutoramento; porém as transições vêm sendo realizadas sem perder de vista os cuidados arrolados com a editoração da Psicologia em Estudo. Os novos membros sempre são professores da graduação e pós-graduação do Departamento de Psicologia da Universidade Estadual de Maringá, tendo já participado de elaborações anteriores, como consultores ad hoc ou mesmo como assistentes de editoração.

Doutora em Educação Escolar, Docente do Departamento de Psicologia da Universidade Estadual de Maringá, Editora da revista Psicologia em Estudo, Brasil.

\# Doutoranda em Desenvolvimento de processos no programa de pós-graduação de Engenharia Química da UEM, membro da comissão executiva da revista Psicologia em Estudo, Brasil. 
Vale ressaltar que em 2011 a revista também obteve recursos do Programa de Apoio a Publicações Científicas do Conselho Nacional de Desenvolvimento Científico e Tecnológico (CNPq) e da Fundação Araucária de Apoio ao Desenvolvimento Científico e Tecnológico do Paraná, o que muito auxiliou na manutenção da qualidade dos procedimentos editoriais. Desta forma, neste último triênio a revista foi contemplada nos dois editais.

Seguindo ainda com a retomada histórica, vale destacar que desde o ano de 2001, a partir do volume 6, número 2 , a revista passou a disponibilizar seus artigos na íntegra pelo Sistema Scielo de publicação (Scientific Electronic Library Online) e na base de dados Scopus Elsevier. Também está indexada em bases de dados referenciais que contêm os dados essenciais dos artigos, como: LILACS (Literatura LatinoAmericana e do Caribe em Ciências da Saúde), do Centro Latino-Americano e do Caribe de Informações em Ciências da Saúde; Index Psi Periódicos, resultante de parceria entre o Conselho Federal de Psicologia e a Pontifícia Universidade Católica de Campinas; CLASE (Citas Latinoamericanas En Ciencias Sociales y Humanidades), gerida pela UNAM (Universidad Nacional Autónoma de México); Sociological Abstracts (Cambridge Scientific Abstracts); Social Services Abstracts, e; Linguistics \& Language Behavior Abstracts. Em 2011 a revista conseguiu três novas indexações DOAJ - Diretory of Open Access Journals (administrado e parcialmente financiado pela biblioteca da Universidade de Lund, da Suécia, financiado pelo Open Society Institute, Biblioteca Nacional da Suécia, SPARC,
SPARC Europe y Axiell), Latindex (Sistema Regional de Informação em Linha para Revistas Científicas de América Latina, o Caribe, Espanha e Portugal) e Psicodoc (Base de dados bibliográfica de Psicologia - Espanha). Além disso, foi encaminhada a documentação para a indexação no REDALYC (Rede de Revistas Científicas da América Latina e no Caribe, Espanha e Portugal).

Considerando a trajetória da revista Psicologia em Estudo em relação à avaliação pelo Qualis de Periódicos, na área de Psicologia, realizada pela comissão conjunta Capes-Anpepp (Coordenação de Aperfeiçoamento de Pessoal de Nível Superior/Associação Nacional de Pesquisa e PósGraduação em Psicologia), importa dizer que em 2005 o periódico foi classificado como Nacional A. Em 2007, a partir da análise dos números editados em 2005 e 2006, a revista foi classificada como Internacional A. Em 2008 os critérios elaborados por essa comissão foram revistos, passando-se a dar grande relevância à indexação dos periódicos em bases de dados reconhecidas internacionalmente, tendo sido as publicações hierarquizadas em estratos (A1, A2; B1, B2, B3, B4, B5) e a revista Psicologia em Estudo foi classificada no estrato A2 e manteve tal classificação na última avaliação do triênio 2009 a 2011.

Com o objetivo de informar aos leitores e colaboradores, destaca-se que o processo de arbitragem dos manuscritos submetidos à Psicologia em Estudo pauta-se no critério de revisão cega (Double blind review) por pares da comunidade científica, ou seja, tanto as identidades de autores como as de assessores ad hoc mantêm-se incógnitas. A tramitação editorial desta revista está resumida na figura 1 . 


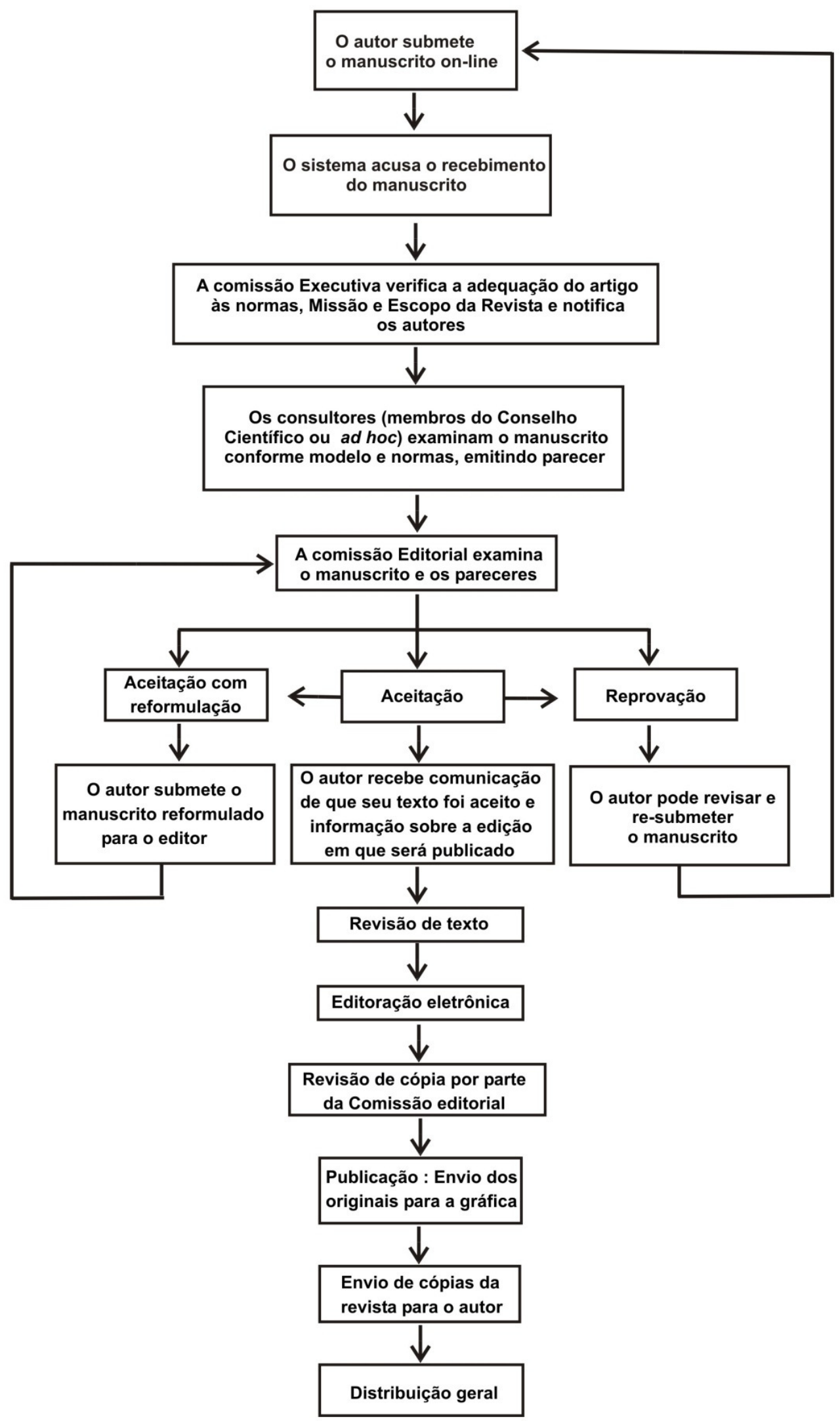

Figura 1 - Fluxograma de tramitação de artigos. 
Este periódico contempla ainda, em suas edições, textos de autores de diferentes universidades nacionais e internacionais, além de profissionais de outras instituições, e tem como objetivo principal incentivar a produção e a divulgação de conhecimentos referentes à Psicologia e áreas afins, promovendo o intercâmbio pedagógico e científico entre professores, acadêmicos e profissionais.

A análise dos dados referentes ao trabalho editorial de 2011, efetuada entre o final de dezembro do mesmo ano e janeiro de 2012, revelou a tramitação de um total de 310 artigos on-line, incluindo-se os pendentes de 2008 (um) artigo, publicado em 2011), 2009 (43 artigos - 32 arquivados e 11 publicados) e 2010 (101 artigos 35 arquivados, 41 publicados, 24 em processo de avaliação e um aprovado para publicação. Em 2011, dos 163 artigos submetidos pelo sistema online, 88 foram arquivados, três estão aprovados para publicação (em edição), 12 foram publicados e até o momento 62 encontram-se em avaliação por consultores.

Na figura 2 é possível verificar a distribuição dos 310 artigos que tramitaram em 2011, nas modalidades Relato de pesquisa, Revisão de literatura, Relato de experiência profissional, Resenha, Seção especial e Entrevista, podendo-se constatar que aproximadamente 55\% deles foram de relatos de pesquisa.

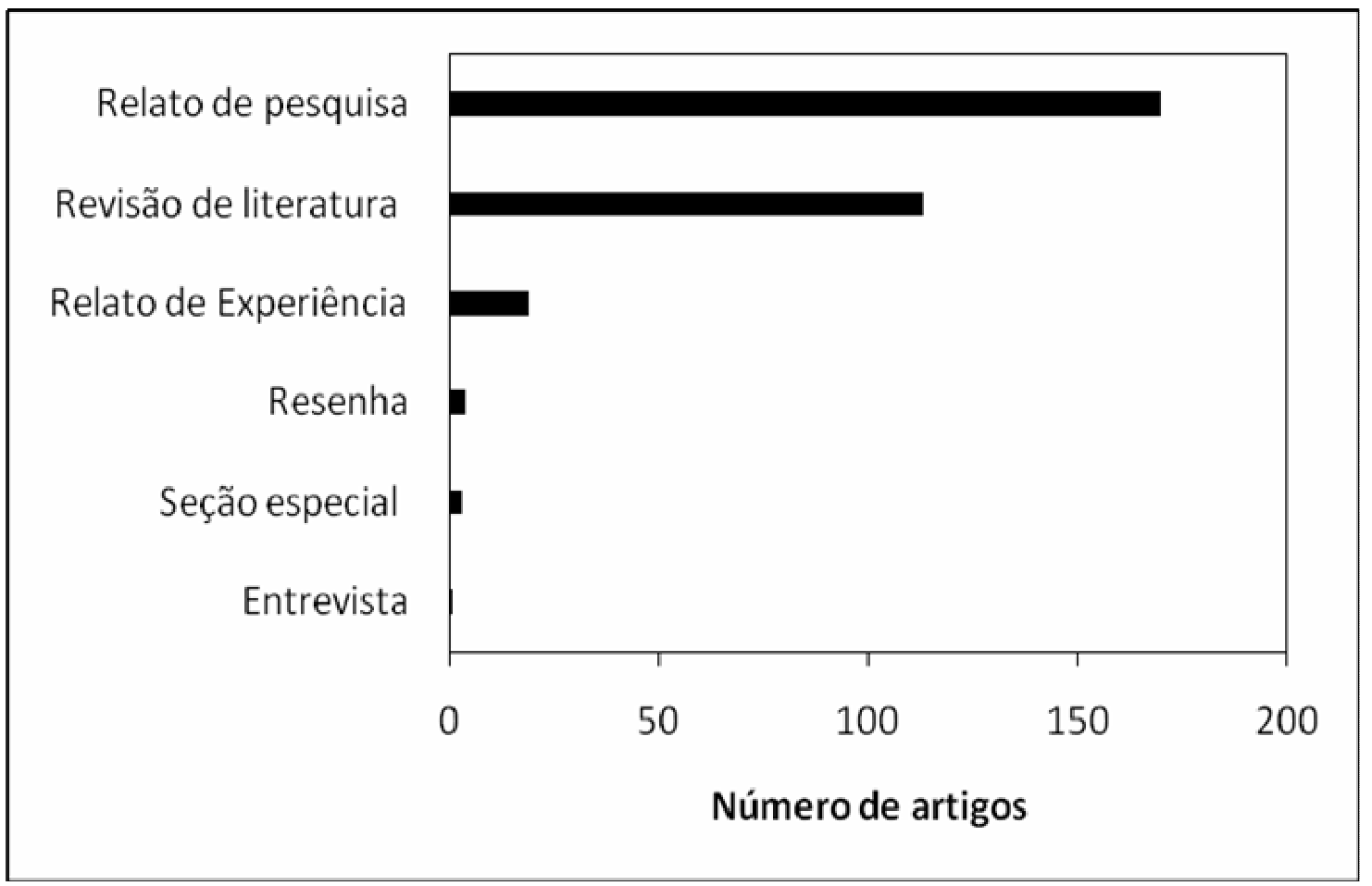

Figura 2 - Modalidade dos artigos que tramitaram em 2011.

A tramitação de artigos teve a colaboração de autores internacionais e nacionais, com predomínio de autores brasileiros. Como as que mais contribuíram com artigos aparecem as regiões Sudeste e Sul do País, e entre estas, o Estado de São Paulo foi o que apresentou o maior número de artigos tramitados (figura 3 ). 


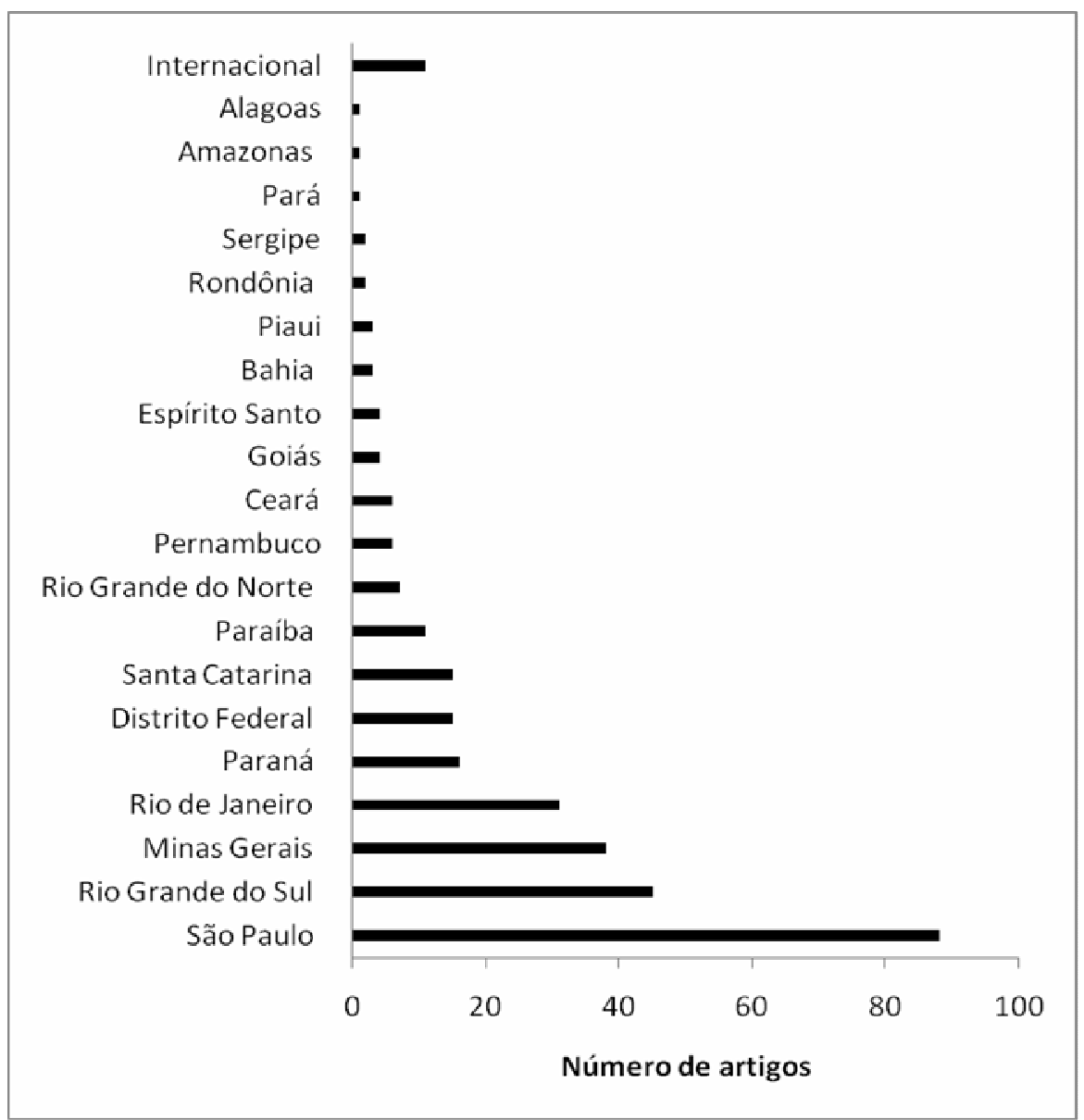

Figura 3 - Regiões donde provieram os artigos que tramitaram em 2011.

De acordo com a instituição de filiação dos autores nacionais, a submissão de artigos de universidades públicas predominou sobre a das privadas (figura 4), o que também se observa em outros periódicos. Além disso, destaca-se a necessidade de implementação da submissão de artigos internacionais, que ainda são recebidos em menor número, se comparados aos artigos nacionais.

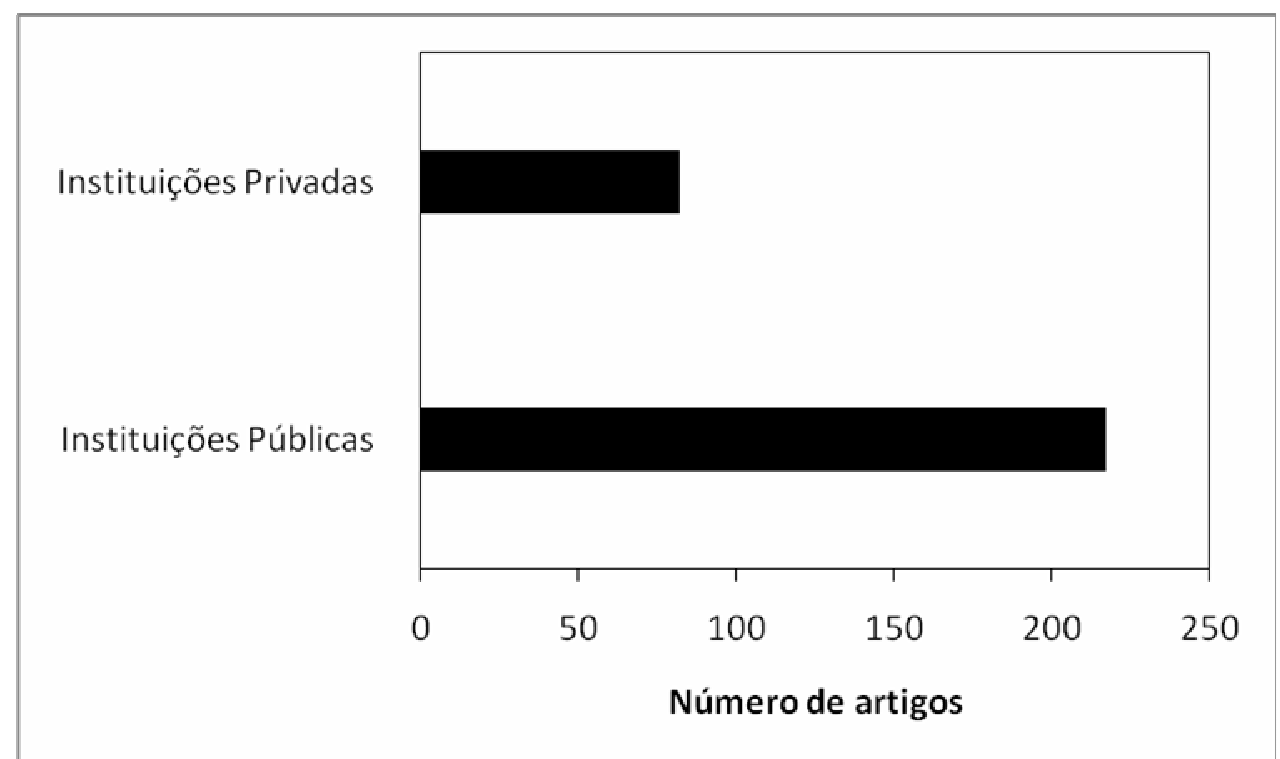

Figura 4 - Vinculação institucional dos autores dos artigos nacionais que tramitaram em 2011. 
No ano de 2011 foram publicados mais artigos submetidos em 2010, objetivando a diminuição da quantidade de artigos de anos anteriores que, ao se acumularem, tendem a aumentar o intervalo entre submissão e publicação, conforme pode ser verificado na Figura 5.

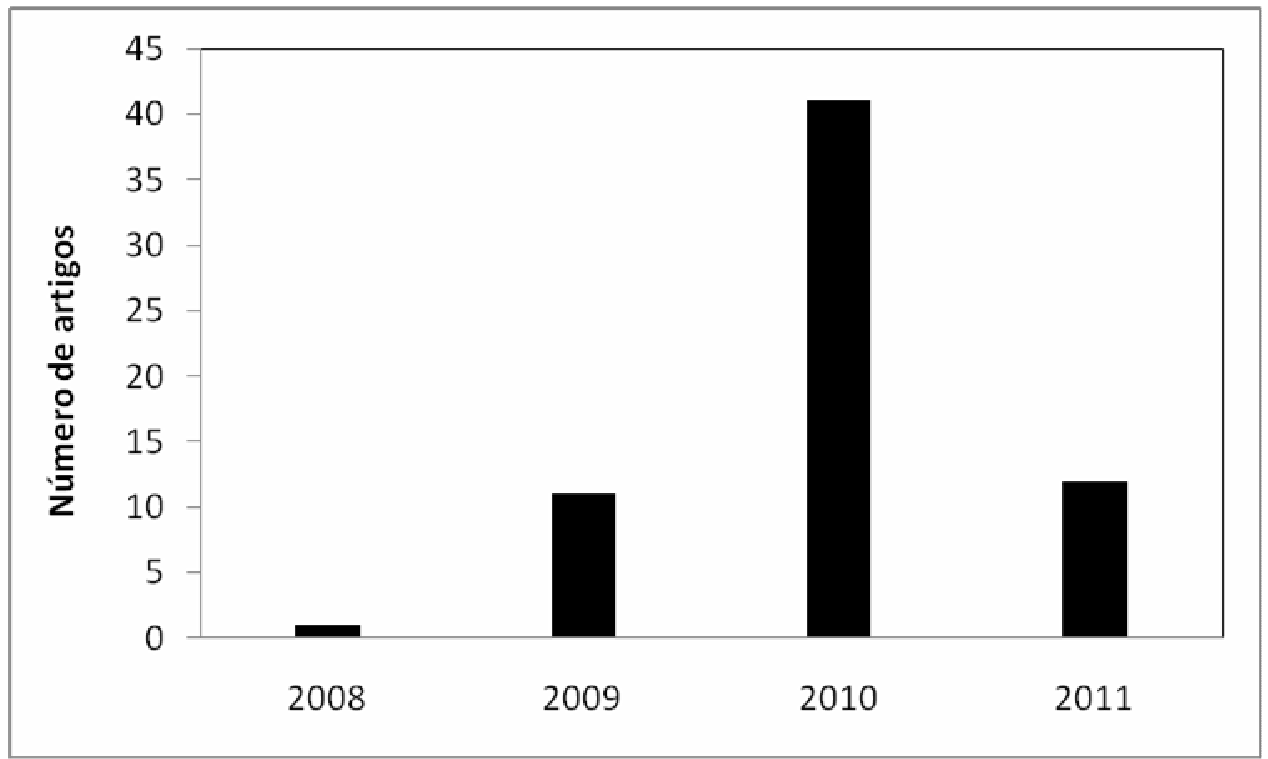

Figura 5 - Número de artigos publicados em 2011.

Do total de 310 artigos que tramitaram no sistema on-line em 2011, foram publicados, ao todo, 65 artigos, dos quais 34 são relatos de pesquisa, 25 são de revisão de literatura/estudo teórico, dois artigos são relatos de experiência profissional, dois da Seção especial, uma resenha e uma entrevista (figura 6), além dos editoriais, que não entram no cômputo geral, por sua natureza diversa. Pode-se constatar que o maior número de artigos publicados em 2011 (59 artigos) foi de relatos de pesquisa/estudos teóricos.

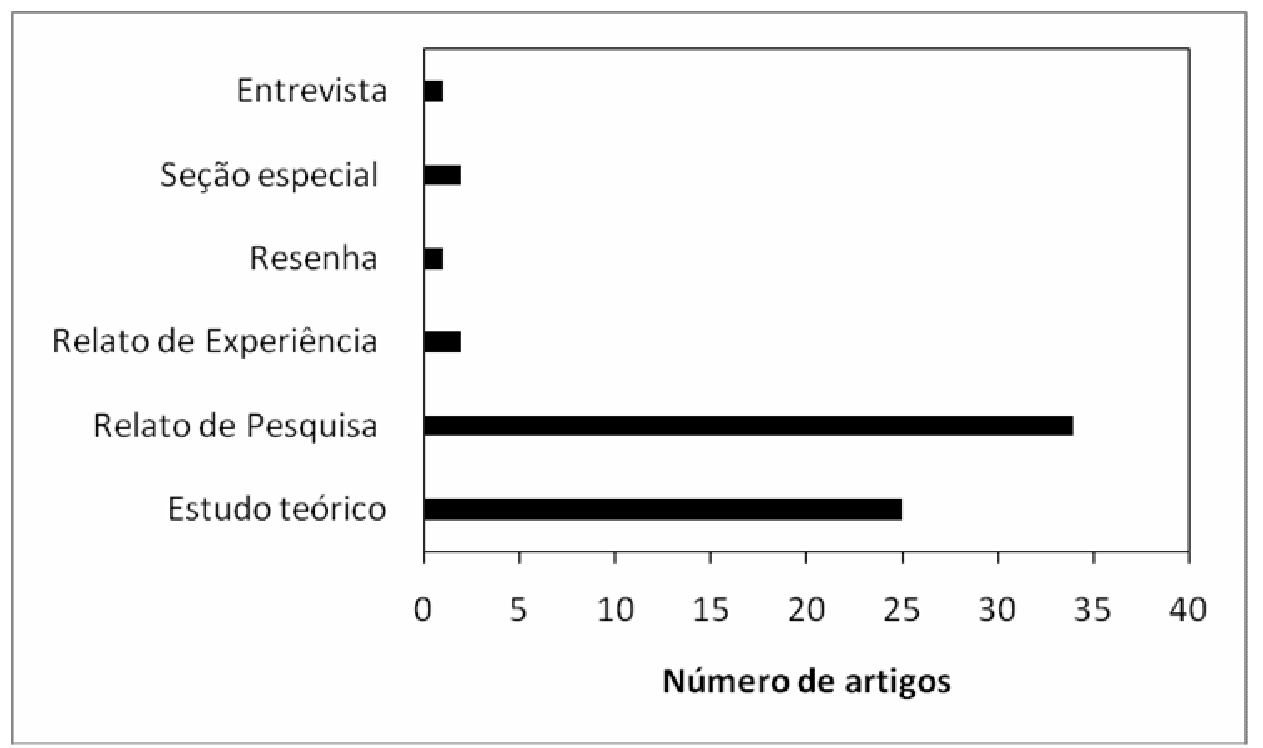

Figura 6 - Modalidades dos artigos publicados em 2011.

Quanto à distribuição dos artigos publicados por região do Brasil, houve predomínio da publicação de artigos provenientes do Estado de São Paulo, vindo em seguida o Rio Grande do Sul e Minas Gerais. Evidencia-se uma evolução na quantidade de artigos de outras regiões e internacionais, no tocante aos publicados em 2011. Houve a predominância da publicação de artigos oriundos de pesquisa e estudos vinculados a universidades públicas (ver figuras 7 e 8 ). 


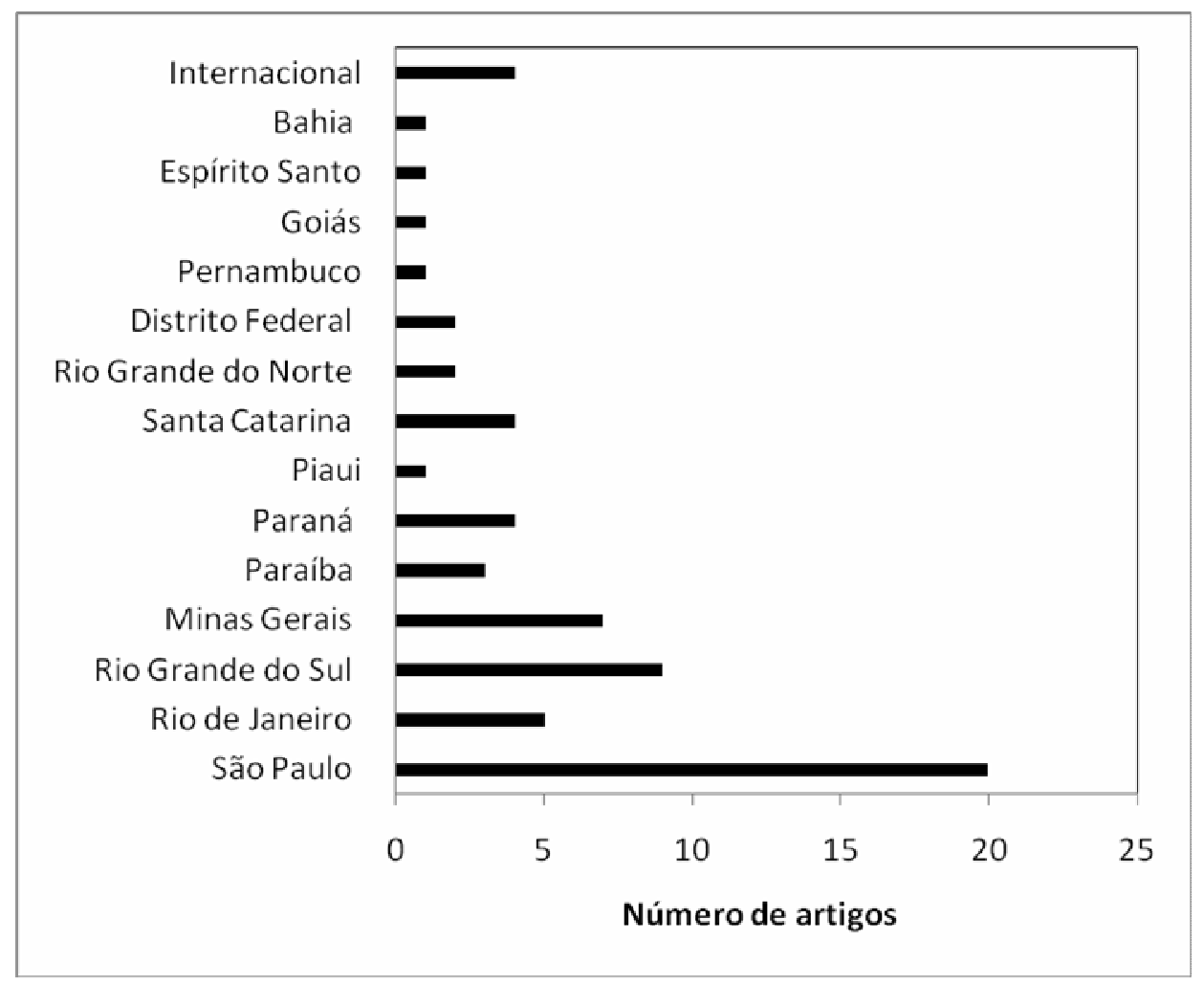

Figura 7 - Regiões dos artigos publicados em 2011.

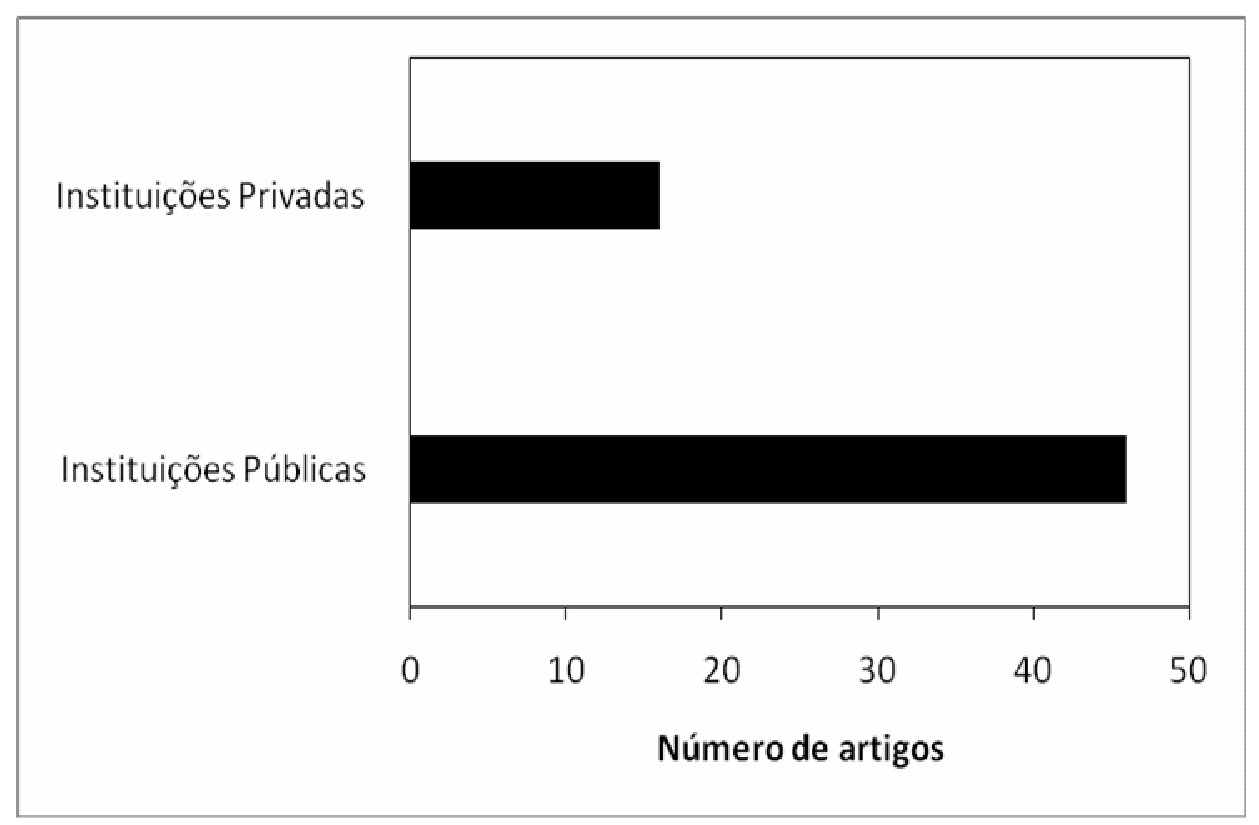

Figura 8 - Vinculação institucional dos autores dos artigos nacionais publicados em 2011.

Em 2011 foi possível reduzir drasticamente os artigos pendentes e acumulados de anos anteriores. Por outro lado, não obstante esse resultado ter sido extremamente positivo para a gestão do periódico, ainda foi mantido um longo intervalo entre submissão e publicação (figura 9), chegando a 15 meses o intervalo entre submissão e publicação; porém estima-se que esta média cairá em 2012, justamente em decorrência de que os artigos que seguem tramitando neste ano são resultantes apenas das submissões de 2011. 


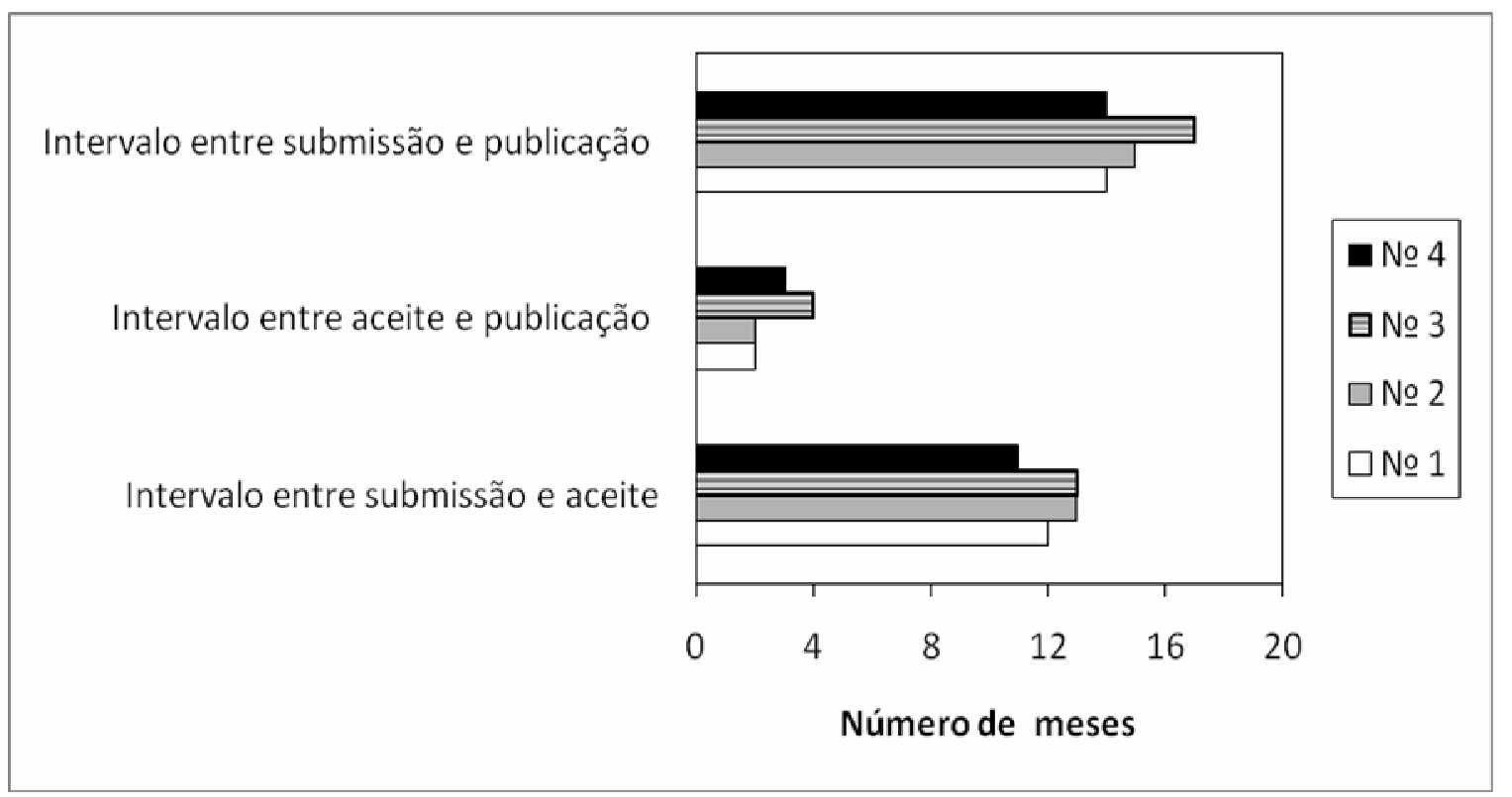

Figura 9 - Período de tramitação dos artigos publicados no volume 16 e seus referentes números.

É importante relatar que em 2011 foi necessário reduzir o número de artigos por número, de uma média de 21 artigos até 2010 para 16, em decorrência do aumento dos custos de impressão da revista sem o correspondente aumento dos repasses de recursos por parte das instituições de fomento. Deste modo, se por um lado a equipe executiva vem se esforçando para reduzir o intervalo entre submissão e publicação, visando escoar o máximo de artigos publicáveis, por outro, a insuficiência de recursos impede que se avance mais na eliminação de artigos pendentes, em função da necessidade financeira posta em 2011, de reduzir a quantidade de artigos por número.

Entende-se que o aumento das submissões de artigos à revista Psicologia em Estudo é fruto de sua visibilidade nacional e internacional e da excelente avaliação que o periódico vem obtendo junto à Capes/Anpepp; porém, contraditoriamente, o aumento da demanda ocasiona dificuldades crescentes à vazão dos artigos aceitos para publicação, o que exige da Equipe Executiva a criação de mecanismos para a seleção dos artigos que tramitarão no periódico.

Um dos procedimentos adotados para a redução do acúmulo de artigos de anos anteriores - acúmulo que compreende o prazo entre submissão e publicação - foi estabelecer suspensões temporárias para novas submissões no sistema on-line. Outra ação adotada pela Equipe Executiva foi uma maior exigência em relação aos artigos submetidos no que tange à sua adequação às normas editoriais e à missão e escopo da revista, bem como em relação à qualidade e originalidade desses artigos. Entre 2010 e 2011, por exemplo, o periódico suspendeu por seis meses as submissões, objetivando readequar a missão, tornando-a mais clara e objetiva, para aperfeiçoar procedimentos como formulários para os avaliadores, elaborar checklist inicial para análise dos artigos assim que são submetidos ao sistema. Com isso foi possível reduzir a demanda e o acúmulo de artigos de um ano para o outro, que vinha ocorrendo anteriormente.

Manter a qualidade do periódico e a avaliação positiva junto ao QUALIS, contudo, não é tarefa fácil, por isso as contradições e problemas enfrentados merecem ser mencionados. Um deles é que, com a passagem de quadrimestral a trimestral a partir de 2008 - que aumentou o custo anual do periódico - obteve-se a publicação de uma média de 21 artigos por número, totalizando a publicação de 84 artigos por volume. O aumento na quantidade de artigos publicados deveu-se à necessidade de dar vazão a artigos acumulados de anos anteriores. Em 2009, por exemplo, a revista ainda publicou artigos de 2006 a 2008. Em 2010 obteve-se uma redução significativa dos artigos pendentes, de modo que em 2011 apenas seis artigos de 2009 permaneceram ainda em tramitação. Isto demonstrou que os mecanismos criados a partir de 2010 pela Equipe Executiva 
trouxe uma redução do saldo de anos anteriores, o que fazia aumentar o intervalo entre a submissão e a publicação dos artigos. No entanto, na mesma velocidade em que a equipe trabalhava para impedir novo acúmulo de artigos pendentes, o número de submissões tendeu a crescer até 2009 , diminuindo a partir de 2010 em virtude das suspensões periódicas das submissões. Como exemplo do exposto, conforme a estatística do SEER, em 2008 a revista teve 108 artigos submetidos, em 2009 teve 190, em 2010 foram 154 e em 2011 foram 163.

Como exposto, um dos procedimentos adotados para frear o aumento crescente de submissões - que, em última instância, poderia ocasionar acúmulo de artigos -, foi estabelecer suspensões temporárias para novas submissões no sistema on-line. Com as suspensões temporárias foi possível evitar o crescente aumento das submissões sem a devida contrapartida no aumento da carga horária dos editores.

Outra ação adotada pela equipe executiva foi a maior exigência em relação aos artigos submetidos, no que tange à sua adequação às normas editoriais e à missão e escopo da revista, bem como à qualidade e originalidade dos próprios artigos. Para corrigir as distorções observadas a Equipe Executiva reformulou a missão do periódico e as normas de orientação aos autores, procurando delimitá-las e deixá-las ainda mais claras. A partir destas reformulações foi elaborado um checklist inicial a ser utilizado pelos editores de seção que fazem a análise preliminar dos artigos submetidos, visando dar maior rapidez ao processo. Tal exigência ocasionou um aumento significativo de artigos arquivados em 2010-2011, em função da não adequação às normas editoriais.

Conforme o relatório emitido pelo Sistema de Editoração Eletrônico (SEER) da revista, em 2011, o índice de arquivamento de artigos foi, em média, de 40\%. Em 2011, o motivos que mais levou ao arquivamento foi rejeição pelo Conselho Editorial, como pode ser constatado na figura 10 . Isto se deve ao aprimoramento realizado pela equipe da revista no tocante à revisão inicial dos artigos quanto às normas técnicas e gramaticais, bem como em relação à consonância com a missão do periódico.

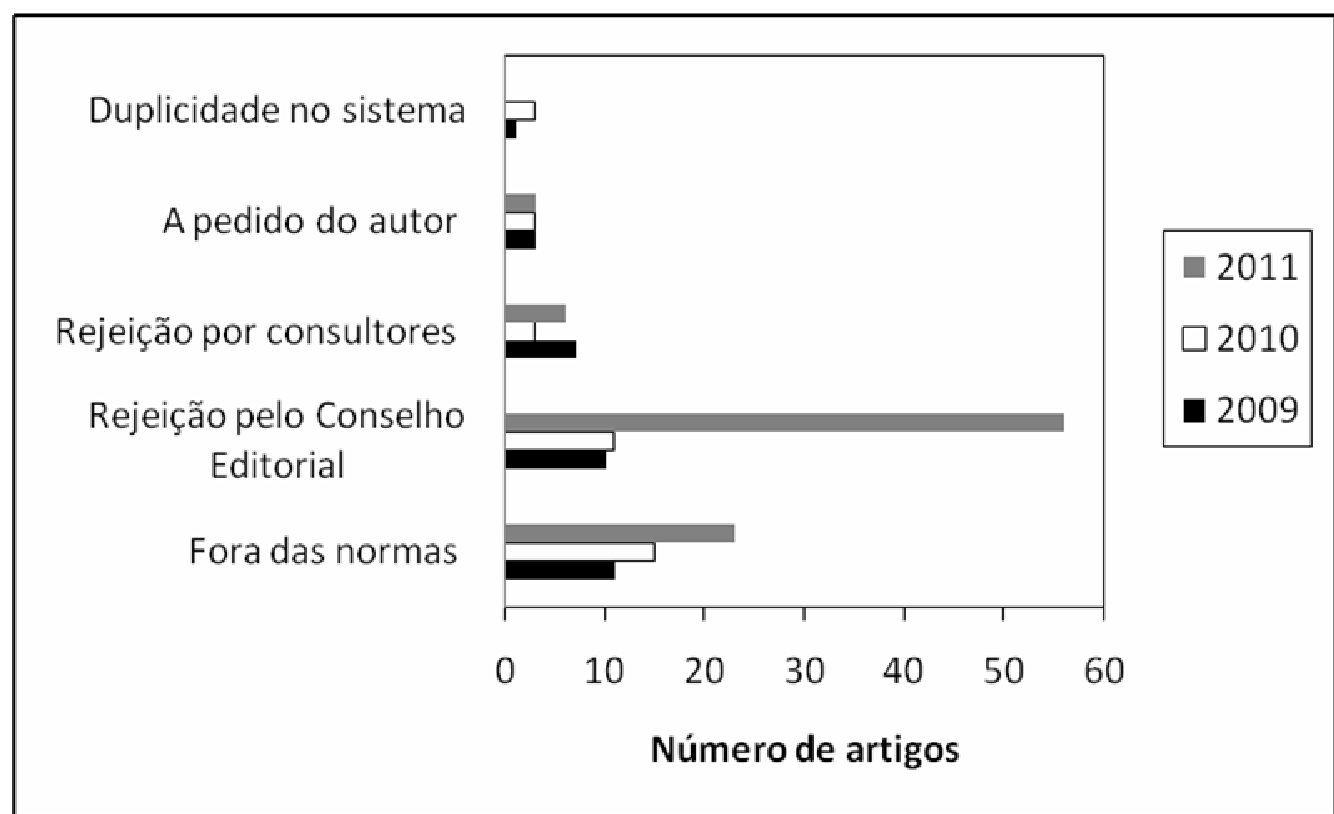

Figura 10 - Número de artigos submetidos em 2009, 2010 e 2011, em relação aos motivos de arquivamento em 2011.

Entre os artigos submetidos em 2011 e 2010 que foram arquivados, predominaram os de Relato de pesquisa, e entre os artigos submetidos em 2009 arquivados em 2011, predominou a modalidade
Revisão de literatura/Estudo teórico (figura 11). Tal como nos artigos publicados, com relação à região, também houve maior arquivamento dos artigos oriundos de São Paulo (figura 12). 


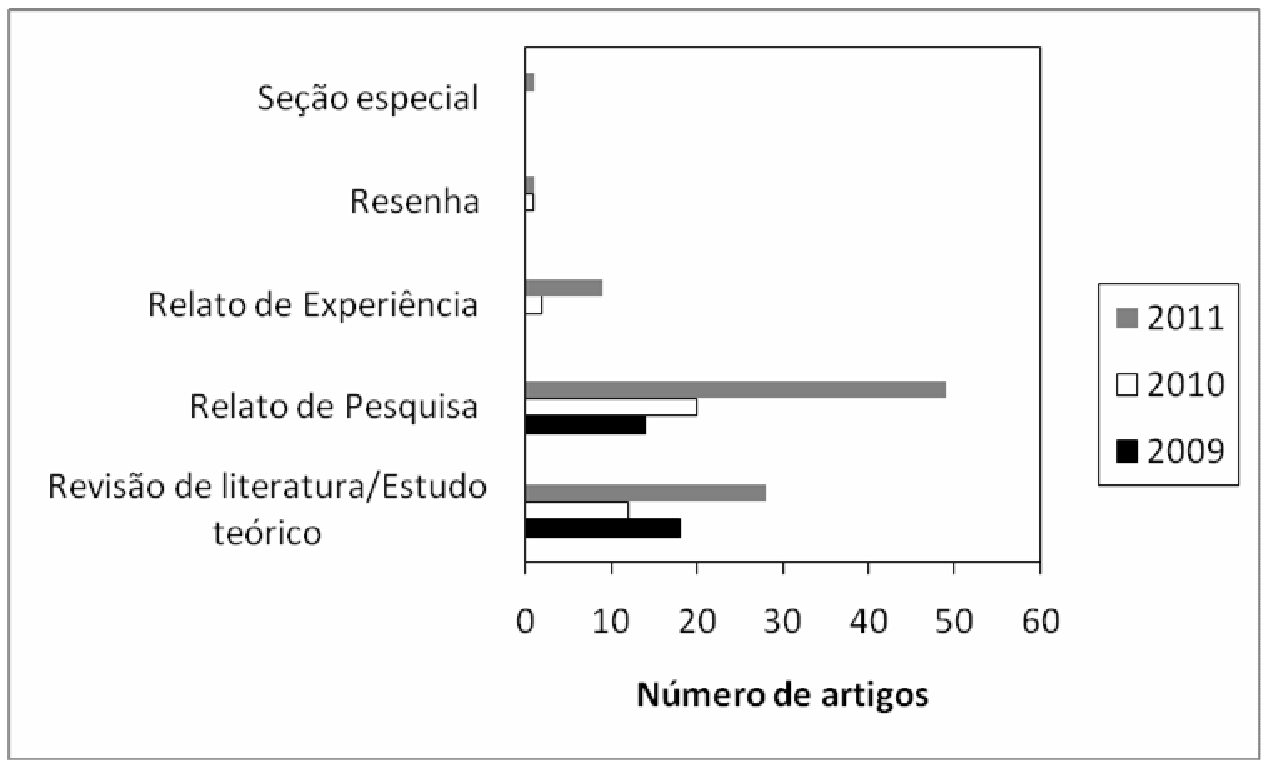

Figura 11 - Modalidade dos artigos submetidos em 2009, 2010 e 2011 que foram arquivados em 2011.

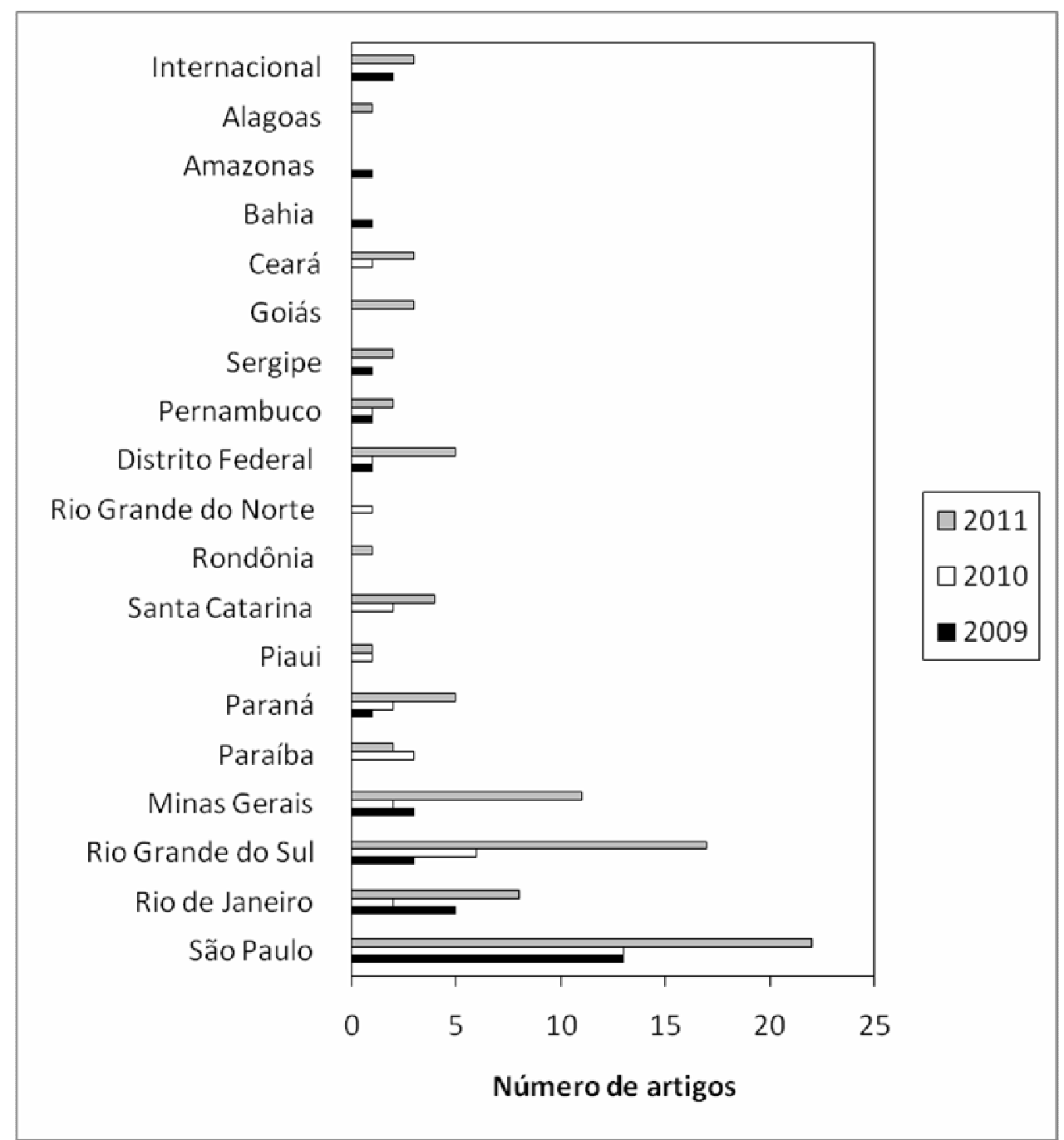

Figura 12 - Regiões dos artigos submetidos em 2009, 2010 e 2011 que foram arquivados em 2011. 
Como aspecto positivo, vale ressaltar que desde 2010, diante das solicitações realizadas pela Equipe Executiva, a revista Psicologia em Estudo obteve apoio estrutural fundamental da Universidade Estadual de Maringá. Hoje conta com uma nova sala e equipamentos de informática adequados para maior agilidade dos processos editoriais. Possui os serviços de uma secretária realocada de outro setor, em regime de 40 horas, e dois bolsistas com 20 horas, o que tornou mais ágeis os procedimentos administrativos relativos à gestão do periódico. Além de tal estrutura administrativa, a revista tem a colaboração dos alunos de pós-graduação (Mestrado em Psicologia) e da graduação em Psicologia da Universidade Estadual de Maringá.

Especificamente em 2011, a revista contou com a colaboração de seis alunos da pós-graduação em Psicologia, distribuídos em diversas funções estratégicas, como análise inicial dos artigos, com referência às normas, organização e preparação de novas indexações, organização, manutenção e controle de permutas nacionais e internacionais, além de dois alunos da graduação em Psicologia que, em conjunto com os acadêmicos da pós-graduação, muito contribuíram para os expedientes internos do periódico.

Como forma de divulgação a revista Psicologia em Estudo conta ainda com um sistema de distribuição do periódico que compreende permutas, doações, vendas e assinaturas, funcionando da seguinte forma: doação de um exemplar de cada número para as IESs públicas que oferecem o curso de Psicologia; permutas de periódicos classificados com conceitos $\mathrm{A}, \mathrm{B}$ e C em âmbito nacional, podendo ser de IESs públicas ou privadas; vendas, por assinatura ou de números avulsos, às IESs privadas que não têm como estabelecer permuta com o nosso periódico; doação de dois exemplares aos autores de cada artigo publicado; doação de um exemplar de cada número para indexadores, membros do Conselho Editorial e dos conselhos de Psicologia; e cortesias esporádicas em eventos científicos da área de Psicologia.

O Conselho Editorial do periódico é composto de 19 profissionais reconhecidos de universidades nacionais e internacionais, que participam das discussões sobre a dinâmica da editoração, quando solicitados. Além disso, a revista Psicologia em Estudo possui um banco de dados de consultores $a d$ hoc cujo número de cadastrados chegou ao total de 1.027 nomes em 2011, a maioria com máxima titulação acadêmica - doutores e pós-doutores - de Universidades de diferentes Estados da federação brasileira, bem como internacionais. Estes profissionais se dispõem a ler e reler (no caso de reformulações), avaliar e emitir um parecer técnico/teórico sobre os textos que recebemos e desta forma prestar um importante serviço para garantir a qualidade de nossa publicação. No final deste relatório será apresentada a nominata dos consultores ad hoc que foram nossos colaboradores em 2011.

Em 2011 outras ações foram implementadas objetivando a divulgação do periódico, como: a participação no $9^{\circ}$ Fórum de Extensão e Cultura da UEM, no período de 10 a 12 de agosto de 2011, apresentando o Painel intitulado "Editoração de Periódico Científico na Área de Psicologia: a experiência de quinze anos da revista "Psicologia em Estudo"; a participação no Congresso Brasileiro de Extensão Universitária, em Porto Alegre, no período de 08 a 11 de novembro de 2011, com a exposição "Revista Psicologia em Estudo: apresentação da experiência de 15 anos de editoração"; e, o envio do artigo científico intitulado A agonia dos periódicos brasileiros: entre o florescimento da privatização na ciência e as trincheiras da socialização do conhecimento científico para a Revista UNITAU Revista de Extensão da Universidade de Taubaté e aprovado para publicação no final de 2011.

Mesmo com os aspectos positivos acima apontados, não foram poucas as dificuldades enfrentadas. Uma delas se refere ao atraso na liberação do recurso pela Fundação Araucária, devido à moratória quando da transição do Governo Estadual, a qual impactou significativamente a regularidade dos números neste ano. A título de exemplo, a revista, que vinha mantendo sua regularidade, no momento atual de elaboração do presente relatório tem publicado no Scielo e impresso apenas os números 1 e 2 do volume 16 , sendo que o número 3 está em fase final de diagramação e o número 4 em etapa final de composição da pauta. Outra dificuldade que a equipe da revista vem enfrentando todos os anos é a rotatividade de seus membros e a não liberação dos editores de outras atividades dentro da universidade, o que faz com que cada editor possa destinar uma pequena carga horária semanal aos trabalhos editoriais, em média, apenas quatro horas.

É importante destacar que, não obstante as dificuldades enfrentadas na editoração e publicação em uma universidade pública estadual - no caso, a Universidade Estadual de Maringá, no Interior do Paraná - que muitas vezes não dispõe de verba suficiente para sua manutenção, ainda assim a revista Psicologia em Estudo vem conseguindo manter-se dentro dos requisitos exigidos em nível nacional e internacional. Além disso, a revista privilegia 
publicações que enfatizem o aprofundamento teórico; assim muitos artigos são oriundos de teses e dissertações, dando prioridade às pesquisas qualitativas na área de Ciências Humanas, o que garante também sua intersecção com outras áreas que não a Psicologia.

\section{NOMINATA DE CONSULTORES 2011}

Abílio Costa-Rosa (UNESP - Assis)

Adriane Xavier Arteche (PUC-RS)

Alex Eduardo Gallo (UEL)

Alice Maggi (Universidade de Caxias do Sul)

Almir Tristão Boechat (UFJF)

Aluísio Ferreira de Lima (UFCE)

Ana Cleide Guedes Moreira (UFPA)

Ana Flávia do Amaral Madureira (UNICEUB)

Ana Jacó-Vilela (UERJ)

Ana Maria Benevides-Pereira (UEM)

Anamélia Lins e Silva (UCSAL)

Andrea Moraes (UFRJ)

Andréa Vieira Zanella (UFSC)

Angela Maria Pires Caniato (UEM)

Angela Maria Resende Vorcaro (UFMG)

Anna Paula Uziel (UERJ)

Brígido Vizeu Camargo (UFSC)

Carmem Lúcia Colomé Beck (UFSM)

Carmen Lucia Cardoso (USP)

Cristina Moreira Marcos (PUC-Minas)

Danilo Saretta Veríssimo (Não tem)

Deise Mendes (UERJ)

Doralice Aparecida Paranzini Gorni (UEL)

Eduardo Augusto Tomanik (UEM)

Eliane Domingues (UEM)

Elisa kern de castro (UNISINOS)

Elizabeth Lima (UEM)

Ênio Brito Pinto (SEDES)

Evani Zambon Marques da Silva (Tribunal de Justiça de São

Paulo)

Fernanda Bittencour Ribeiro (PUC-RS)

Gilcinéia Rose da Silva Santos (CESUMAR)

Henriette Tognetti Penha Morato (USP)

Henrique Figueiredo Carneiro (UFPB)

Isabel Cristina Vasconcelos de Oliveira (UNIFOR)

Jacqueline Moreira de Oliveira (PUC-MG)

Jadson Justi (UCDB)

Janaína Bianca Barletta (UFSE)

Jorge Luís Ferreira Abrão (UNESP-Assis)

Juliana Pasqualini (UNESP-Bauru)

Leda Mariza Fischer Bernardino (USP)

Léia Priszkulnik (USP)

Lenita Gama Cambaúva (UEM)

Lígia Márcia Martins (UNESP)

Lucia Cecília da Silva (UEM)
Lucinéia Maria Lazaretti (UFSCAR)

Ludgleydson Fernandes de Araújo (UFPI)

Luiz Rohden (UNISINOS)

Marcela Mansur-Alves (UFMG)

Marcelo Luis Grassi Beck (UNEMAT)

Marco Antonio de Castro Figueiredo (USP-Ribeirão Preto)

Maria Alves Bruns (FFCLRP-USP)

Maria Cláudia Santos Lopes de Oliveira (UNB)

Maria Cristina de Oliveira *não foi encontrado

Maria Cristina Marquezine (UEL)

Maria Cristina Moreno Matias (UEL)

Maria da Graça Correa Jacques (UFRGS)

Maria da Graça Correa Jacques (UFRGS)

Maria Dalva Santos Alves (UFC)

Maria de Fátima Belancieri (USC)

Maria de Fátima Souza Santos (UFPE)

Maria de Jesus Dutra dos Reis (UFSCAR)

Maria do Carmo Borges de Souza (UFRJ)

Maria Elizabeth Barros de Barros (UFES)

Maria Lúcia Miranda Afonso (Centro Universitário UMA-MG)

Maria Regina Maciel (UERJ)

Maria Teresa Castelo Branco (UFPR)

Maria Teresa Lisboa Nobre Pereira (UFES)

Mariane Lima de Souza (UFES)

Marilda Gonçalves Dias Facci (UEM)

Marina de Bittencourt Bandeira (UFSJ)

Mário Sérgio Ribeiro (UFJF)

Marisa Todescan da Silva Baptista (UNIMARCOS)

Marta Chaves (UEM)

Mary Sandra Carlotto ( Universidade Luterana do Brasil-Canoas)

Meyre Eiras de Barros Pinto (UEL)

Paulo Cesar Seron (UEM)

Andréia Isabel Giacomozzi (Secretaria Municipal de Saúde de Florianópolis)

Raquel Martins de Assis (UFMG)

Regina Célia Souza Brito (UFPA)

Regina Perez Christofolli Abeche (UEM)

Reinaldo Furlan (USP)

Ricardo Azevedo da Silva ( PUC-Pelotas)

Ricardo Ottoni Vaz Japiassu (UNEB)

Roberto Bello (UFRJ)

Rozilda das Neves Alves (UEM)

Sandra Francesca Conte de Almeida (UCB)

Sheila Giardini Murta (UNIB)

Sheila Regina de Camargo Martins (UEM)

Sonia Alberti (UERJ)

Stella Regina Taquette (UERJ)

Tales Vilela Santeiro (UFGO-Jataí)

Tânia dos Santos Alvarez Silva (UEM)

Tania Rudnicki (ULB)

Tereza Cristina Cavalcanti (UnB)

Tonantzin Ribeiro Gonçalves (UFRGS)

Verônica Lucia do Rego Luna (UFPB)

Viviana Carola Velasco Martinez (UEM) 